\title{
Olfactory cleft opacity and CT score are predictive factors of smell recovery after surgery in nasal polyposis*
}

C. Vandenhende-Szymanski, B. Hochet, D. Chevalier, G. Mortuaire

Department of Otorhinolaryngology and Head and Neck surgery, Huriez Hospital, University of Lille, France
Rhinology 53: 29-34, 2015

DOl:10.4193/Rhino 14.160

*Received for publication:

July 4,2014

Accepted: September 21, 2014

\begin{abstract}
Objectives: To assess subjective improvement of olfactory function following endoscopic sinus surgery (ESS) in chronic rhinosinusitis associated with nasal polyps (CRSwNP) and to analyse factors of recovery with the European Test of Olfactory Capabilities (ETOC).
\end{abstract}

Methods: We carried out a prospective study of 30 patients with CRSwNP from November 2011 to April 2013. The ETOC was filled the day before surgery and in the short term follow-up. Sixteen suprathreshold odorants with a detection task and a forced choice verbal identification task were tested.

Results: The mean composite score (MCS) improved at 3 and 6 months. The preoperative MCS was correlated to the Lund-Mackay score and to the olfactory cleft opacification on preoperative computed tomography (CT) scan. Multivariate linear regression modelling of patients with preoperative anosmia showed that the olfactory recovery at 3 months was predicted by the preoperative Lund-Mackay score and the age, and at 6 months by the preoperative Lund-Mackay score.

Conclusion: With a convenient psychophysical test, we showed that olfactory cleft opacification and CT scan score could be predictive factors of olfaction disorder severity and improvement after ESS in CRSWNP. These results need to be strengthened in the long term with a larger panel of patients.

Key words: olfaction, nasal polyposis, endoscopic sinus surgery, olfactory test, anosmia

\section{Introduction}

With nasal obstruction, olfactory disorders in Chronic Rhinosinusitis with Nasal Polyps (CRSwNP) are the most prominent symptoms with a negative impact on the quality of life (1). When corticosteroid treatments are inefficient to relief those symptoms, Endoscopic Sinus Surgery (ESS) is often offered with rare but potential risks of skull base and eye-socket injuries. Regarding olfaction and its improvement after surgery, giving clear information on symptoms outcomes is often difficult ${ }^{(2)}$. Several studies, based on each patient's self-reported symptoms or quantitative assessment, have described a clinical improvement of the olfaction after ESS ${ }^{(3,4)}$.
Many tools for olfactory function assessment have been developed. Most of them are psychophysical devices based on the presentation of odours and the recording of the patients' response. While screening tests only differentiate between normal and pathological states, more extensive tests allow for a reliable discrimination between anosmic, hyposmic and normosmic subjects. Better tests have to be based on normative data acquired and validated in large samples ${ }^{(5)}$. The Sniffin'Sticks test is widely used with three assessment methods of olfactory function (n-butanol odour threshold, odour discrimination and odour identification) ${ }^{(6)}$. More recently, the European Test of Olfactory Capabilities (ETOC-CNRS UMR5292 France) has been 
proposed. Built as a cross-cultural tool, the ETOC is designed to evaluate both detection and identification capabilities ${ }^{(7)}$. It allows classification of subjects in three groups: anosmic, hyposmic or normosmic.

Herein, we proposed to evaluate the ETOC on a homogeneous population of CRSWNP patients to assess feasibility of the test, to measure olfactory outcomes after surgery and to determine clinically-relevant predictors of olfactory function after surgery.

\section{Material and Methods}

\section{Study setup}

A prospective study was conducted from November 2011 to April 2013 including patients with CRSwNP. The diagnosis of CRSwNP was established according to the EACCI criteria ${ }^{(8)}$. The patients were addressed in our department for persistent symptoms despite optimal medical treatment (at least 3 courses of 7-day oral corticosteroid and maximum dose of topical nasal corticosteroid spray). Concomitant asthma (diagnosed by spirometry), allergy (confirmed with skin prick testing) and history of acetylsalicylic acid (ASA) intolerance were assessed. Adults ( $\geq 18$ years old) patients were enrolled after maximum medical management failure and EES indication. Patients with immunodeficiency, autoimmune disease and/or cystic fibrosis were excluded. A procedure of endoscopic ethmoidectomy was scheduled for each patient. It was a radical anterior and posterior ethmoidectomy with middle turbinate resection and sphenoidotomy. To improve the homogeneity of the population, any kind of medical therapy (oral and topical nasal corticosteroid) except nasal douching was stopped at least one month before surgery. An informed consent was obtained from each patient.

The day before surgery, a computed tomography (CT) scan was performed to evaluate the olfactory cleft opacification (Figure 1) and the Lund-Mackay score (maximum total score: 24$){ }^{(9)}$. The size of the olfactory cleft was evaluated on CT scan millimetre coronal and parasagittal planes on computer-assisted surgery workstation (Digi-pointeur ${ }^{\circledast} 6200$, Collin) by two independent
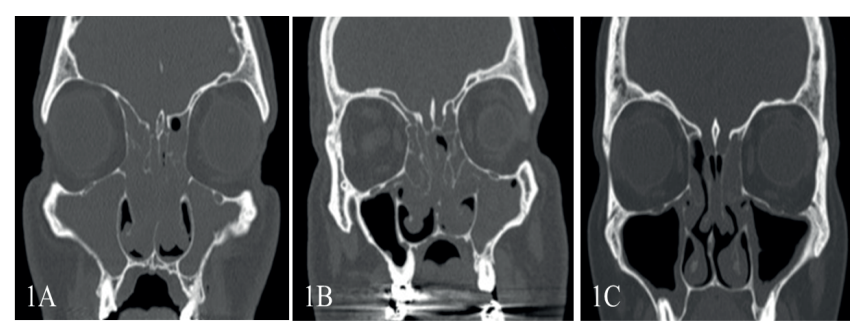

Figure 1. CT scan assessment of the olfactory cleft opacification. Bilateral (A), unilateral (B) opacification or no opacification (C) of the olfactory clefts were noticed on CT coronal plane between the root of each middle turbinate vertical lamella and the nasal septum.
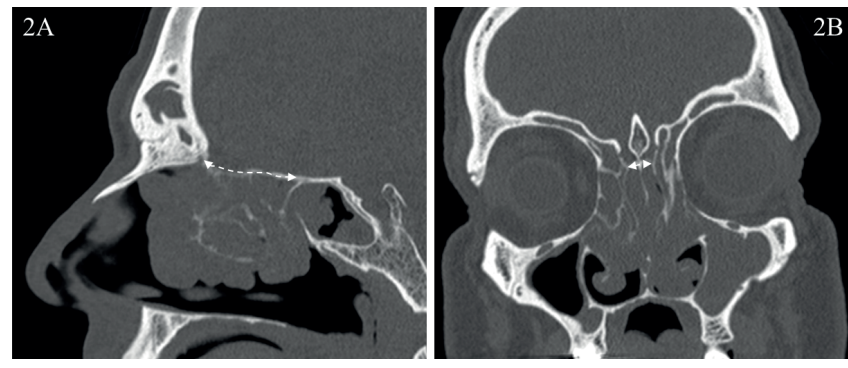

Figure 2. CT scan measurement of the size of the cribriform plate. A) The length of the cribriform plate was measured on parasagittal CT plane between the frontal sinus posterior wall and the spheno-ethmoidal roof junction. B) The width was measured on CT coronal plane between the roots of the two middle turbinate vertical lamellae.

investigators (CVS, GM). Contrast, brightness and image angles could be adjusted on the workstation to improve bony details. This was particularly useful in significantly diseased sinuses. The length of the cribriform plate was measured from the frontal sinus posterior wall to the spheno-ethmoidal roof junction (Figure 2A). The width was measured between the roots of the two middle turbinate lateral lamellae (Figure 2B). The polyps size was endoscopically measured using a 30-degree rigid nasal fiberoptic scope according the Lildholdt grading system (0-absence of polyps, 1-polyps in the middle meatus only, 2-polyps beyond the middle meatus but not reaching the inferior edge of the inferior turbinate, 3-polyps completely obstructing the nose) ${ }^{(10)}$. The ETOC was performed the day before surgery, at 3 months and 6 months postoperatively. To avoid misinterpretation of the results, all the patients were treated after surgery with the same topical nasal corticosteroid (mometasone fuorate) at the same

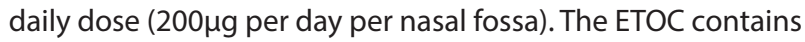
16 series of 4 vials blocks. In each block, only one vial contains a supra-threshold odorant. The first part of the test consists of a detection task. The patient tells which vial of the block contains the odorant. The second part of the test is an identification task. Once the right vial has been identified, the patient is asked to choose the right descriptor between 4 proposed. This procedure allows to restrain the number of correct responses by chance and to detect cheaters. A detection score and an identification score are added to obtain a mean composite score (MCS) ranging from 0 to 32 . Three groups of patients are described: anosmic (MCS $<10$ ), hyposmic (MCS between 10 and 26), normosmic (MCS $\geq 27$ ).

\section{Statistical analysis}

Data were input into Microsoft Exce ${ }^{\circledR}$ and statistical analysis was performed with SPSS ${ }^{\circledR}$ v 15.0 statistical software (SPSS Inc., Chicago, IL, USA). The non-parametric Wilcoxon test was used to compare the means of paired samples. The non-parametric Mann-Whitney and Kruskal-Wallis tests were used to compare 
Table 1. Comparison of the preoperative Mean Composite Score according to preoperative clinical characteristics and CT scan data.

\begin{tabular}{|lc|}
\hline Preoperative Data & p-value \\
\hline Age $^{1}$ & 0,67 \\
\hline $\begin{array}{l}\text { Delay between CRSwNP } \\
\text { diagnosis and EES }\end{array}$ & 0,59 \\
\hline Cribriform plate size & \\
\hline Lund-McKay CT score $^{1}$ & 0,28 \\
\hline Gender & $0,038$ (rho $=-0,38)$ \\
\hline Concomitant asthma & \\
\hline allergy $^{2}$ & 0,41 \\
\hline ASA intolerance $^{2}$ & 0,12 \\
\hline Prior polypectomy $^{2}$ & 0,8 \\
\hline Olfactory cleft opacification $^{3}$ & 0,09 \\
\hline
\end{tabular}

${ }^{1}$ Pearson correlation test; ${ }^{2}$ Mann-Whitney test; ${ }^{3}$ Kruskal-Wallis test

the means of non-paired samples. Correlation analyses were conducted with the Pearson test (using rho as correlation coefficient). A linear regression model was built to identify potential prognosis factors of olfactory recovery. The outcome of interest was the 3-month and 6 month-postoperative changes (postoperative minus preoperative) in ETOC score. Means, standard deviations (SD) or ranges are reported for descriptive statistics. A two-tailed $p$-value $\leq 0.05$ was considered statistically significant.

\section{Results}

Thirty CRSwNP patients were included ( 21 male/9 female) with a mean age of 44 years (range: 18-68). The mean delay between the diagnosis of CRSWNP and the surgical procedure in our department was 12 years (range: 1-35). Twelve patients (40\%) had a past history of endoscopic polypectomy. On endoscopy examination, the mean polyp size was 2.4 (range: 1-3). Thirteen patients (43\%) had concomitant asthma, 12 patients (40\%) had allergy, and 4 patients (13\%) had ASA intolerance. No environmental exposure (living conditions, workplace and smoking) was noticed.

The mean CT scan score was 19.2 (range: 11-24). There was bilateral olfactory cleft opacifications in 11 cases (36.7\%), unilateral opacification in 5 cases (16.7\%) and no opacification in 14 cases (46.6\%). The length and the width of each cribriform plate measured independently by the 2 investigators of the study (CVS, GM) were correlated (length: $r h o=0.91, p=0.001$; width: rho $=$ $0.975, p=0.001)$. The mean total surface of the cribriform plate (both sides included) was $232 \mathrm{~mm}^{2}$ (SD $45.8 \mathrm{~mm}^{2}$ ).

All 30 patients completed the preoperative and postoperative ETOC with a mean completion time of $15 \mathrm{~min}$. Mean preoperative MCS was 9.9 (range: 0-27, SD 8.5). Among them, 19 were anosmic (MCS < 10) (63\%), 9 were hyposmic (MCS between 10 and 26 ) (30\%) and 2 were normosmic (MCS $\geq 27$ ) (7\%). The preoperative MCS was analysed by comparison with clinical and CT data (Table 1). Preoperative MCS was better in patients without prior polypectomy (MCS without prior polypectomy $=12.9$, MCS with prior polypectomy $=5.5 ; \mathrm{p}=0.027$ ). Preoperative MCS was inversely correlated with the preoperative CT scan score (rho = $-0.4 ; p=0.038$ ). Preoperative MCS was better in patients without preoperative olfactory cleft opacity on CT scan $(p=0.037)$. Over-

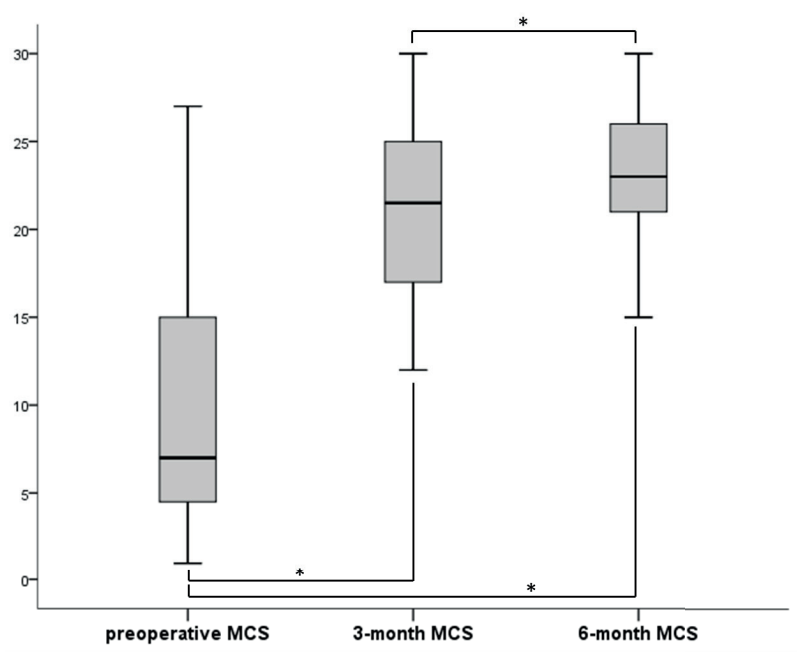

Figure 3. Improvement of the Mean Composite Score after ESS.

* Significant improvement of the MCS at 3 months $(p=0.0004)$, at 6 months $(p=0.0002)$ and between 3 and 6 months $(p=0.037)$.

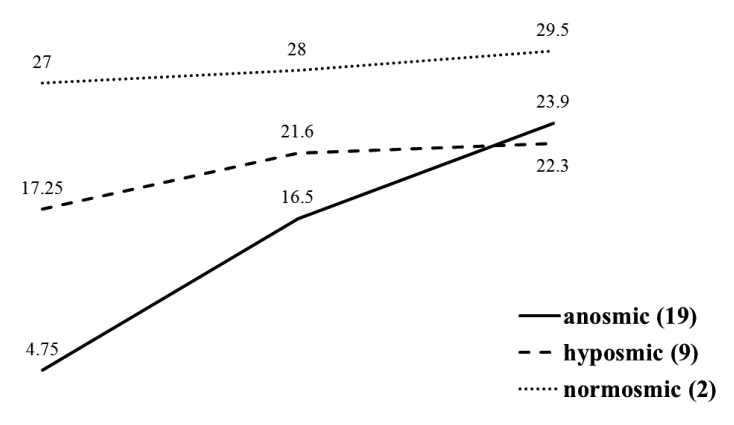

Preop-MCS

3 month-MCS

6 month-MCS

Figure 4. Olfaction recovery after ESS with ETOC according to the preoperative olfactory status of the patients.

Every preoperative MCS-anosmic patient (19) was improved at 3 months and 6 months. No preoperative MCS-hyposmic (9) or MCS-normosmic (2) patient was worsened at 3 months and 6 months. 
all, the 3-month MCS $(19.2$, SD 6.5) was improved ( $p=0.0004)$. The 6-month MCS (22.4, SD 5.2) was improved by comparison with the preoperative MCS ( $p=0.0002)$ and the 3-month MCS $(p=0.037$ ) (Figure 3). No hypo- or normosmic patient in the preoperative period was altered at 3-month and 6-month postoperative follow-up (Figure 4). Mainly, the 19 preoperative anosmic patients improved their status at 3 months in 14 cases (13 became hyposmic, 1 normosmic, 5 remained anosmic). One of these 5 anosmic patients at 3 months became hyposmic at 6 months. It is noteworthy that preoperative hypo- and normosmic patients did not show substantial improvement of their olfactory status after surgery (Figure 4). No statistical difference in MCS scores was observed in the postoperative period for preoperative hyposmic patients.

Multiple linear regression model was used to identify statistical links between the olfactory outcomes (at 3 months and 6 months) and the data collected in the preoperative period for the 19 patients with preoperative anosmia (MCS < 10). A descendant substantive knowledge method was used (backward selection). Through this approach, we showed that prognosis of olfactory recovery at 3 months (difference between preoperative MCS and 3-month $M C S$ ) was negatively influenced by the preoperative CT scan score $(p=0.011)$ and the age of the patient $(p=0.008)(R$-squared $=0.48)$. At 6 months, only the $C T$ scan score had a negative impact $(p=0.024)$.

\section{Discussion}

Olfactory disorders in CRSwNP are a major concern for the patients. They do often complain with a nagging doubt about its recovery after medical treatment or ESS. The etiology of olfactory dysfunction in CRSWNP involves both conductive and sensorineural mechanisms ${ }^{(11)}$. Lane et al. showed in a model of olfactory inflammation that chronic induction of proinflammatory cytokines expression exposed axonal bundles to macrophage-mediated molecular damage ${ }^{(12)}$.

Many testing procedures have been developed in the literature to assess odour perception. Based on odour identification with threshold measurement, most of those tests circumvent the olfactory-verbal gap that frequently separates an odour from its name and show great culture dependence ${ }^{(7)}$. Moreover, the threshold determination procedure could be time-consuming for both subjects and experimenters. Like in the Sniffin'Sticks test that includes a discrimination procedure to assess the olfaction by different tasks, the ETOC measures separately above-threshold detection and identification to provide separate information on the processes of "peripheral" perception and "central" recognition.

Herein we have shown that this test was rapid and easy to use in the assessment of the olfactory disorders in chronic rhinosi- nusitis. In our study, the preoperative analysis of the olfactory status was established at least 1 month after oral corticosteroid wash-out. This design allowed a comparison of the patients' status on clinical and CT scan data. Only 2 out the 30 patients were normosmic before surgery. This result underlines the burden of dysosmia in CRSwNP patients. Several studies about the olfaction of patients with advanced nasal polyposis have noticed that less than $18 \%$ of the patients were normosmic with variable tests (Olfactory Threshold Test, Smell Identification Test, Sniffin'Sticks Test) ${ }^{(13,14)}$. Olfaction disability in CRSwNP could be partly explained by a conductive disorder. Severe thickness and oedema of the nasal mucosa with large polyp size limit the passage of odourant molecules towards their receptors ${ }^{\left({ }^{8}\right)}$. Thus, a negative correlation was observed between the preoperative MCS and the CT scan score in our study. Moreover opacifications of the olfactory cleft before surgery were associated with a worse preoperative MCS. These findings corroborate with the study of Hong et al. showing a relation between olfactory disability and olfactory cleft opacifications in 106 patients with chronic rhinosinusitis ${ }^{(15)}$. Alike, Kim at al. described a correlation between olfactory cleft opacification and postoperative olfactory results ${ }^{(16)}$. In a subsequent study, the same authors showed that preoperative olfactory cleft opacification was predictive of bad olfactory results after surgery, even in patients with low 6-month postoperative Lund-Mackay score ${ }^{(17)}$. Concerning the size of the olfactory cleft measured on CT scan, we did not find a correlation with the preoperative MCS. This critera has not been analyzed in the literature. Among the others factors recorded in the preoperative assessment, only prior surgery was significantly associated with a poor preoperative MCS in our study. We assume that the remodelling process observed after surgery with concomitant inflammation and adhesions could lead to injuries of the olfactory neuroepithelium with persistent oedema of the adjacent mucosa. Others factors impairing the olfactory status of patients with chronic rhinosinusitis have been reported in literature. Concomitant asthma, current tobacco use, long-standing nasal polyposis have been described as pejorative ${ }^{(18)}$. Age is also a known factor associated with olfactory dysfunction ${ }^{(18,19)}$. Both age-increased olfactory receptor neuron cell death and inhibition of olfactory neurogenesis by inflammation may have a synergestic effect over time in elderly patients with CRSwNP ${ }^{(18,20)}$.

Overall, the 3-month MCS and the 6-month MCS were significantly improved. No patient suffered from a deterioration of the olfactory score during the short term follow-up. We assume that preoperative hypo- and normosmic patients did not exhibit significant change after surgery as their preoperative score was relatively high to observe any further improvement. Among the 19 anosmic patients in the preoperative period, $73.5 \%$ of them improved their olfaction at 3 months. This result corro- 
borates with Delank et al. showing an improvement of $70 \%$ of the patients after functional ESS ${ }^{(21)}$. ESS enables the odorants to reach the olfactory cleft, it promotes mucous drainage and opens the gate through which topical nasal steroids can reduce inflammation near the nasal neuroepithelium. It is also noteworthy that the resection of the middle turbinate has no effect on the olfactory recovery in our study. Jankowsky et al. described the same result underlining that the human olfactory neuroepithelium is located predominantly on the dorsal aspects of the nasal vault, the septum, and the superior turbinate ${ }^{(22)}$. In a functional investigation of the olfactory epithelium distribution, Leopold and al. showed by means of electro-olfactogram and located biopsy specimens that the olfactory sensitive region was extended anteriorly and superiorly to the middle turbinate insertion ${ }^{(23)}$. In a randomised trial including 1,106 matched CRS patients with and without polyps, Havas et al. did not observe complications specific to middle turbinate resections ${ }^{(24)}$. Similar results were obtained on olfactory recovery by Soler et al. with a non randomised prospective study ${ }^{(25)}$.

The last objective of our study was to delineate predictive factors of olfactory recovery after ESS in CRSwNP. With a logistic regression model applied on patients with a preoperative anosmia (MCS $<10$ ), we showed that preoperative CT scan score and the age were associated with a poor recovery at 3 months in preoperative anosmic patients. At 6 months, only the CT scan score was significantly concerned. These findings are consistent with the role of both conductive and sensorineural processes in the olfaction of patients with CRSwNP. We assume that patients with high CT scan scores exhibit more inflammation near the nasal neuroepithelium. As EES must circumvent this area, the surgical removal of the polyps is insufficient to relieve oedematous mechanical obstruction of the olfactory cleft. Moreover, age-related dysfunction persists after ESS, limiting the impact of the surgery on the process of recovery. Others studies have tried to define predictive factors of olfactory recovery after ESS. The use of different types of objective olfactory measures makes interpretation of the data difficult, leading to different outcomes. Inclusion of patients without polyps, patients with preoperative corticotherapy or patients with different surgical management (septoplasty, polypectomy, ethmoidectomy associated with corticosteroid treatment) adds confusion to the interpretation of the results ${ }^{(3)}$. Jiang et al. showed that the olfactory improvement was not predicted by the extent of the disease on CT scan in a study including both patients with or without polyps and patients with allergic rhinitis ${ }^{(26)}$. In a multivariate linear regression analysis of Litvak et al. including patients with or without polyps (with a subset of them taking topical and/or systemic corticosteroids), baseline olfactory status (namely, anosmia) and nasal polyposis were both significant predictors of improvement with the Smell Identification Test score at 12-month postoperative follow-up ${ }^{(27)}$. Like in our study, others variables including gender, asthma, allergy, ASA intolerance, history of prior surgery were not significant variables in their model (27). In others studies, duration of olfactory deficit and previous sinus surgery presented significant predictive value for the short-term outcome of the olfactory function after ESS ${ }^{(14)}$. Smoking, known as a risk factor of olfactory impairment, was not a comorbidity reported by the patients in our cohort.

Our study size may have been a limitation to detect a difference in patients with uncommon comorbidities such as smoking or ASA intolerance. Yet, the homogeneity of our population (only CRSwNP) with a uniform medical management in the preoperative period (1-month withdrawal of oral corticosteroid) and in the postoperative period (same daily dose of topical corticosteroid) strengthen the reliability of the olfactory status comparison. Even if longer follow-up is needed to assess olfactory outcomes, our ETOC findings may be useful for allowing us to counsel our patients more accurately on the likelihood of objective improvement after ESS.

\section{Conclusion}

Olfactory dysfunction in CRSwNP is complex and multifactorial. Both conductive and sensironeural processes are implicated as demonstrated by the role of CT scan score and age in the recovery after EES. The ETOC is a convenient tool in the assessment of olfactory impairment in those patients. Our study shows the impact of the olfactory cleft opacification in nasal polyposis olfaction disorder and a clear improvement after ethmoidectomy in the follow-up. Severe preoperative CT scan scores and age of the patients were negative factors of recovery at 3 and 6 months in preoperative anosmic patients. Importantly, no preoperative normosmic patient became anosmic after the surgery. Further studies are required to determine long term changes that may occur in the late postoperative period.

\section{Authorship contribution}

CVS: data generation and analysis, preparation of the manuscript $\mathrm{BH}$ : data generation

DC: study conception and design

GM: study conception and design, data generation, data analysis and interpretation, preparation of the manuscript.

\section{Conflict of interest}

No conflict to be reported 


\section{References}

1. Mortuaire G, Vandeville S, Chevalier D. Psychometric evaluation of the SinoNasal Outcome Test-16 for quality of life in chronic rhinosinusitis with nasal polyps. Eur Ann Otorhinolaryngol Head Neck Dis. 2010; 127: 91-96.

2. Landis BN, Stow NW, Lacroix JS, Hugentobler M, Hummel T. Olfactory disorders: the patients' view. Rhinology. 2009; 47: 454-459.

3. Pade J, Hummel T. Olfactory function following nasal surgery. Laryngoscope. 2008; 118: 1260-1264.

4. Schriever VA, Gupta N, Pade J, Szewczynska M, Hummel T. Olfactory function following nasal surgery: a 1-year follow-up. Eur Arch Otorhinolaryngol. 2013; 270: 107-111.

5. Hummel T, Konnerth CG, Rosenheim K, Kobal G. Screening of olfactory function with a four-minute odor identification test: reliability, normative data, and investigations in patients with olfactory loss. Ann Otol Rhinol Laryngol. 2001; 110: 976-981.

6. Hummel T, Sekinger B, Wolf SR, Pauli E, Kobal G. 'Sniffin' sticks': olfactory performance assessed by the combined testing of odor identification, odor discrimination and olfactory threshold. Chem Senses. 1997; 22: 39-52.

7. Thomas-Danguin T, Rouby C, Sicard G, et al. Development of the ETOC: a European test of olfactory capabilities. Rhinology. 2003; 41: 142-151.

8. Fokkens WJ, Lund VJ, Mullol J, et al. European Position Paper on Rhinosinusitis and Nasal Polyps 2012. Rhinol Suppl. 2012; (23): 1-298

9. Lund VJ, Mackay IS. Staging in rhinosinusitus. Rhinology. 1993; 31: 183-184.

10. Lildholdt T, Rundcrantz $H$, Lindqvist $N$ Efficacy of topical corticosteroid powder for nasal polyps: a double-blind, placebo-controlled study of budesonide. Clin Otolaryngol Allied Sci. 1995; 20: 26-30.

11. Raviv JR, Kern RC. Chronic sinusitis and olfactory dysfunction. Otolaryngol Clin North Am. 2004; 37: 1143-1157.

12. Lane AP, Turner J, May L, Reed R. A genetic model of chronic rhinosinusitis-associated olfactory inflammation reveals reversible functional impairment and dramatic neuroepithelial reorganization. J Neurosci. 2010; 30: 2324-2329.

13. Litvack JR, Mace JC, Smith TL. Olfactory function and disease severity in chronic rhinosinusitis. Am J Rhinol Allergy. 2009; 23: 139-144.

14. Danielides $V$, Katotomichelakis $M$, Balatsouras D, et al. Evaluation of prognostic factors for olfaction in nasal polyposis treated by endoscopic sinus surgery. Rhinology. 2009; 47: 172-180.

15. Hong SC, Leopold DA, Oliverio PJ, et al Relation between $C T$ scan findings and human sense of smell. Otolaryngol Head Neck Surg. 1998; 118: 183-186.

16. Kim DW, Kim JY, Jeon SY. The status of the olfactory cleft may predict postoperative olfactory function in chronic rhinosinusitis with nasal polyposis. Am J Rhinol Allergy. 2011; 25: 90-94.

17. Kim DW, Kim JY, Jeon SY. Postoperative olfactory results in chronic rhinosinusitis with nasal polyposis according to wound healing status. Clin Exp Otorhinolaryngol. 2013; 6: 146-151.

18. Litvack JR, Fong K, Mace J, James KE, Smith TL. Predictors of olfactory dysfunction in patients with chronic rhinosinusitis. Laryngoscope. 2008; 118: 2225-2230.

19. Doty RL, Shaman P, Applebaum SL, Giberson R, Siksorski L, Rosenberg L. Smell identification ability: changes with age. Science. 1984;226:1441-3.

20. Kern RC. Chronic sinusitis and anosmia: pathologic changes in the olfactory mucosa. Laryngoscope. 2000; 110: 1071-1077.

21. Delank KW, Stoll W. Olfactory function after functional endoscopic sinus surgery for chronic sinusitis. Rhinology. 1998; 36: 15-19.

22. Jankowski R, Bodino C. Olfaction in patients with nasal polyposis: effects of systemic steroids and radical ethmoidectomy with middle turbinate resection (nasalization) Rhinology. 2003; 41: 220-230.

23. Leopold DA, Hummel T, Schwob JE, Hong SC, Knecht M, Kobal G. Anterior distribution of human olfactory epithelium. Laryngoscope. 2000; 110 :417-421.

24. Havas TE, Lowinger DS. Comparison of functional endonasal sinus surgery with and without partial middle turbinate resection. Ann Otol Rhinol Laryngol. 2000; 109: 634-640.

25. Soler ZM, Hwang PH, Mace J, Smith TL. Outcomes after middle turbinate resection: revisiting a controversial topic. Laryngoscope. 2010; 120: 832-837.

26. Jiang RS, Su MC, Liang KL, et al. Preoperative prognostic factors for olfactory change after functional endoscopic sinus surgery. Am J Rhinol Allergy. 2009; 23: 64-70.

27. Litvack JR, Mace J, Smith TL. Does olfactory function improve after endoscopic sinus surgery? Otolaryngol Head Neck Surg. 2009; 140: 312-319.

\section{Geoffrey Mortuaire}

Service d'ORL de chirurgie

cervico-faciale, Hôpital Huriez

CHRU Lille, 59000

France

Tel: +(33)-32-044 5675

Fax: +(33)-32-044 6220

E-mail: g-mortuaire@chru-lille.fr 